\title{
Inferring Market Structure from Customer Response to Competing and Complementary Products
}

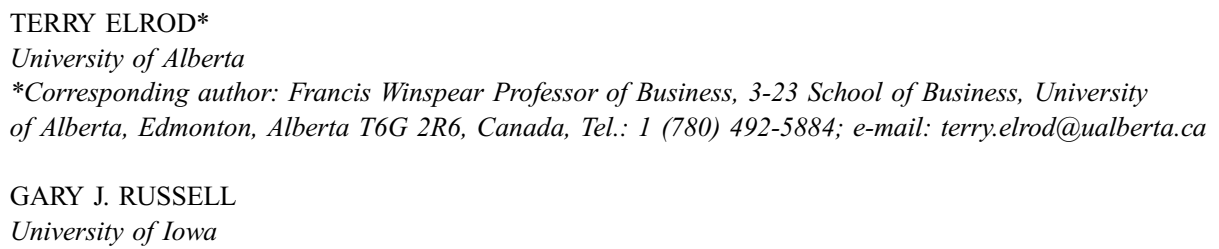




\begin{abstract}
We consider customer influences on market structure, arguing that market structure should explain the extent to which any given set of market offerings are substitutes or complements. We describe recent additions to the market structure analysis literature and identify promising directions for new research in market structure analysis. Impressive advances in data collection, statistical methodology and information technology provide unique opportunities for researchers to build market structure tools that can assist "real-time" marketing decisionmaking.
\end{abstract}

Key words: market structure analysis, competition, complementary products, product class definition

Market structure analysis seeks to explain the nature and extent of competition among companies and their products. Historically, market structure analysis has emphasized either supply-side or demand-side explanations, but not both (Kadiyali, Karunakaran and Rao 2001). Supply-side considerations are discussed in the extensive economic literature on industrial organization. This paper explores how demand-side considerations create distinctive patterns of competition.

We adopt a broad definition of market structure analysis: Market structure analysis explains the extent to which the market offerings under consideration are substitutes or complements. This definition is meant to suggest the following:

Market structure analysis explains. A model of market structure must do more than simply reflect the extent to which pairs of products are substitutes or complements. A primary purpose of market structure analysis is to obtain a fundamental understanding of competition that will be useful even as new offerings are introduced and existing ones are altered or withdrawn.

Market structure is largely determined by customers. Companies decide what offerings to provide and use the marketing mix to inform and influence customers, but sales are determined by how customers perceive and respond to these offerings. Customers are influenced by their own particular situations and experiences (Ratneshwar et al. 1999), and they decide whether and to what extent they substitute one market offering for another (substitutes) or use two offerings in unison (complements).

Customers do more than choose among simple products. When necessary, we emphasize this by referring to market offerings rather than products. A market offering may be a product, service, combination of product and service, or a bundle of products and/or services.

The market structure analyst must specify the set of market offerings to consider. One cannot, in a single analysis, seek to explain the structure underlying competition among all offerings available in the marketplace. Historically, most analyses of market structure have studied choice among brands within a narrowly-defined product category. The rapidly increasing availability of low-cost data, powerful computers, and better 
modeling technologies means that more ambitious market structure analyses are increasingly attractive and possible.

Complementarity also merits investigation. It is surprising that complementarity has been neglected by the market structure analysis literature because many new product offerings combine for customers what they had previously combined for themselves. Such new offerings are bundles of what are, for the customer's specific purpose, complementary products and/or services.

The rest of this paper begins by briefly describing the research tradition in market structure analysis. We then highlight significant recent developments and conclude with recommendations for further research.

\section{The Research Tradition}

Marketers have always been interested in understanding market structure. An important aid to such understanding is provided by multi-attribute utility theory, which recognizes that customers buy products as means to ends rather than as ends in themselves. Customer preference for a product is driven by (i) the attributes of a product and (ii) the value the customer attaches to these attributes. We may expect that customer valuation of a product's attributes will be related to its intended use.

Multi-attribute utility theory assists our understanding of customer purchasing in two important ways: (i) it provides a useful summary of customer perceptions and evaluations of existing products in terms of product attributes, and (ii) at least in theory, it allows prediction of customer purchasing for any combination of new, repositioned and existing products that may be described in terms of these same attributes. Classical treatments of market structure analyses of preference/choice data are of two types: external and internal (Carroll 1972).

External analyses presume that the attributes driving choice, and the values of the brands on these attributes, are known to the researcher. External analysis consists of explaining customer preferences/choices for brands in terms of the importance customers attach to these attributes. The best-known example of this approach is conjoint analysis. An important advantage of conjoint analysis is that it allows the researcher to study customer response to hypothetical (and yet to be introduced), and not just existing, market offerings.

Another important type of external analysis uses data about consumer perceptions of existing brands. While the researcher must decide which attributes to ask customers about, it is customer perceptions of the brands, and not researcher judgment, that determine the values the brands have on these attributes. Often most of the variability in how customers perceive existing brands can be explained using a few dimensions that underlie the many attributes typically included in the survey. A preliminary analysis is used to determine the number of dimensions, the relation of each of these dimensions to the original attributes, and the locations of the brands on these dimensions. The result of such an analysis is commonly referred to as a perceptual map. However, when it comes time to explain 
customer preferences/choices for brands, the perceptual map (along with the locations of the brands in this map) is again taken as given.

Internal analysis of preference/choice data takes a different approach (cf. DeSarbo and Rao 1986; Elrod 1991; DeSarbo, Manrai and Manrai 1993). As in perceptual mapping, it assumes that a few dimensions suffice to explain substitutability/complementarity among brands. But in an internal analysis the number of dimensions, and the locations of the brands on these dimensions, is determined solely from preference/choice data. The dimensions of the resulting map, however, are unlabeled. Managerial judgment and/or a subsequent analysis of consumer perceptions are needed to interpret the map.

External and internal analyses of consumer choice among existing brands each have their advantages. External analyses yield a map that is closely tied to how customers perceive the brands and that adequately accounts for nonrandom variability in customer perceptions. Internal analyses can possibly identify all the important dimensions underlying preference/choice behavior, whether anticipated by the researcher or not, and the brands' locations are scaled to fit the preference/choice data.

In 1997, Sawtooth Software arranged for a direct comparison of the internal and external market structure analysis approaches. These two approaches were applied by different researchers to data collected as part of the same study (Elrod 1997; Wittenschlaeger and Fiedler 1997). This comparison revealed that the two different sources of information about market structure used by these two methods - preferences and perceptions-yielded similar perceptual maps. This result is typical in commercial applications and strongly suggests that data on both perceptions and preferences should be used in a single comprehensive model of market structure (Bacon 2001; Johnson 2001).

\section{Recent Developments}

Recent research is extending the scope and sophistication of market structure analysis. We highlight some of these developments in this section.

\subsection{Inferring Customer Representations of Objective Product Attributes}

At the heart of demand-side market structure analysis is the belief that customers use simplified representations of brands when making choices. Given the substantial empirical support of this belief, it is surprising that little provision for such simplifications has been made in the conjoint analysis literature. Some notation will help clarify recent investigations of this idea.

Let $\mathbf{X}_{i}$ be the $N \times K$ matrix that describes the $N$ conjoint profiles presented to the $i$ th customer in terms of $K$ attributes, and let $\mathbf{y}_{i}$ be a vector of this customer's ratings of these profiles. Typically, the customer's preference ratings are explained using the linear regression model

$$
\mathbf{y}_{i}=\boldsymbol{\alpha}_{i}+\mathbf{X}_{i} \boldsymbol{\beta}_{i}+\boldsymbol{\epsilon}_{i}
$$


where $\boldsymbol{\beta}_{i}$ is the importance that the $i$ th customer attaches to each of the $K$ attributes. Typically $\mathbf{X}_{i}$ is a dummy-variable coding of all levels of all attributes and $K$ is large, which means (1) implies customers formulate their brand preferences using high-dimensional multi-attribute utility functions.

Suppose instead that customers view and evaluate the profiles in $M \ll K$ dimensions, or "benefits." This may be modeled linearly as

$$
\boldsymbol{\beta}_{i}=\mathbf{A} \boldsymbol{\gamma}_{i} \text { for all } i,
$$

where $\mathbf{A}$ is a $K \times M$ matrix that relates the $K$ attributes to the $M$ underlying benefits, and $\boldsymbol{\gamma}_{i}$ is an $M$-element vector that characterizes the $i$ th customer's preferences for these $M$ benefits.

Lenk (2001) used (1) and (2) to reanalyze the ratings-based conjoint data from Lenk et al. (1996). He found that a single dimension accounted for consumer ratings of personal computer systems, one that reflected the trade-off between low price and system speed, power and functionality. This model was more in agreement with the data than the more general model that allowed $\boldsymbol{\beta}_{i}$ to vary over $i$ in all $K$ dimensions.

Simplifications less stringent than (2) are also possible. Elrod and Häubl (1998) investigated

$$
\begin{aligned}
& \boldsymbol{\beta}_{i}=\boldsymbol{\mu}+\mathbf{A} \boldsymbol{\gamma}_{i} \text { and } \\
& \boldsymbol{\beta}_{i}=\boldsymbol{\mu}+\mathbf{A} \boldsymbol{\gamma}_{i}+\boldsymbol{\zeta}_{i},
\end{aligned}
$$

where $\zeta_{i}$ is distributed multinormal with zero mean and diagonal dispersion. They found, consistently across three data sets, that (4) performed better than allowing $\boldsymbol{\beta}_{i}$ to vary in $K$ dimensions, while (3) performed worse.

\subsection{Clustering Perceptual Data and Diagnosing Competitive Relationships}

Cluster analysis is very familiar to marketers. It is frequently used to partition customers into a small number of segments. The advantages are both computational and conceptual. Clusters allow managers to think in terms of perhaps a dozen or fewer customer "types" rather than of thousands or even millions of individual customers. Typically, cluster analysis is applied to demographic, lifestyle and/or behavioral data for a sample of customers. To be useful, the clusters must prove in subsequent analyses that they adequately represent the variability in customer perceptions or preferences.

Mazanec and Strasser (2000) discuss in detail a modern alternative to cluster analysis as applied to customer perceptions of brands. They consider binary perceptual data, which is easy to obtain for many attributes and brands. They employ the topology representing network (TRN) of Martinetz and Schulten (1994), which may be used, as is the more familiar k-means clustering algorithm, to identify segments possessing minimum variance. The TRN possesses several advantages relative to k-means cluster analysis: (i) it is better at finding a minimum-variance solution, particularly given large data sets; (ii) it reports the extent to which each pair of clusters is proximate (i.e., close together) in attribute space; 
and (iii) it is capable of detecting intricate (e.g., non-convex) cluster structures not easily captured by conventional methods.

Mazanec and Strasser (2000) apply the TRN to binary perceptual data by having it categorize each customer's ratings of each brand, without informing the algorithm of the identity of the customers or brands. In this way, the researcher can apply exact statistical tests to diagnose inter-brand competition and to determine whether the clusters are significantly related to the brands. Subsequent analyses are necessary to determine the relationship between cluster membership and brand choice. A close relationship is not guaranteed. However, since perceptual data are being analyzed, the clusters invariably provide a good characterization of customer differences in how they perceive the brands.

\subsection{Simultaneous Analysis of Perceptions and Preferences}

In 1999, Sawtooth Software introduced composite product mapping (CPM), which estimates customer-specific product maps, based on customer perceptions, that best accounts for customer brand preferences (Sawtooth Software, Inc. 1999). The vector version of CPM has a preference structure identical to (1) and (2), with the $\mathbf{X}_{i}$ in (1) being the $i$ th customer's ratings of $N$ existing brands on $K$ attributes. Substituting (2) into (1) yields

$$
\mathbf{y}_{i}=\boldsymbol{\alpha}_{i}+\left(\mathbf{X}_{i} \mathbf{A}\right) \boldsymbol{\gamma}_{i}+\boldsymbol{\epsilon}_{i} .
$$

The term $\left(\mathbf{X}_{i} \mathbf{A}\right)$ is indeed a customer-specific map of the brands in $M$-dimensions.

Here the maps are estimated simultaneously with the customers' importance weights, and so the maps, although based on the perceptual data, provide the best account for customer preferences. As a result, differences in perceptions related to consumer preference are retained in the map, while differences unrelated to preference are not. This is an important improvement upon external market structure analysis. However, the model cannot detect an attribute that affects preference unless customers were asked to rate the brands on that attribute.

\subsection{Internal Market Structure Analysis-Clusters of Buyers and Brands}

While many models conceive of market structure as maps of brands in a benefits space of few dimensions (e.g., Elrod 1988, Elrod and Keane 1995), others employ tree structures and/or latent clusters (cf. Elrod 1991). Chaturvedi and Carroll's (1994) nonsymmetric version of their SINDCLUS model, which recovers latent clusters, may also be used for internal market structure analysis. Let $\mathbf{Y}_{t}$ be an $N \times J$ matrix describing the purchasing by $N$ customer groups (as predefined by the researcher) for $J$ brands during time period $t$. The $n$th row of $\mathbf{Y}_{t}$ may be the volume shares of the $J$ brands for the $n$th customer group (i.e., its "share of requirements"). The nonsymmetric SINDCLUS model represents $\mathbf{Y}_{t}$ as

$$
\mathbf{Y}_{t}=\mathbf{U S}_{t} \mathbf{B}^{\prime}+c_{t} \mathbf{1 1}^{\prime}+\mathbf{E}_{t},
$$


where $\mathbf{U}$ is an $N \times K$ binary matrix that assigns the $N$ user groups to the $K$ latent clusters, $\mathbf{S}_{t}$ is a diagonal matrix of shares for that time period, $\mathbf{B}$ is a $J \times K$ binary matrix that assigns the $J$ brands to the $K$ latent clusters, $c_{t}$ is a scalar, $\mathbf{1 1}^{\prime}$ is an $N \times K$ matrix of ones and $\mathbf{E}_{t}$ an $N \times K$ matrix of errors.

The model estimates $\mathbf{U}, \mathbf{S}_{t}, \mathbf{B}$ and $c_{t}$ simultaneously for all $T$ time periods. The pattern of assignments of brands and user groups to latent clusters reveals the structure in the market. Chaturvedi and Carroll (2001) applied (6) to one market of 22 brands and 22 user groups, resulting in 10 latent clusters with considerable face validity.

\subsection{Multiple-Item Analysis}

A significant limitation of much work in the market structure area is the assumption that the market consists of a single product category composed only of substitutes. (Cooper (1988) provides a notable exception.) As observed by Shocker, Bayus and Kim (2001), many consumer choice tasks involve products from multiple product categories. A proper market structure analysis of such choice settings must be able to represent complementarity as well as substitutability among items and to make sense of simultaneous purchase of different items (i.e., explicit or implicit bundling). Most models for market structure analysis are designed to study choice among items that compete so closely that single-item purchasing (in which purchase of one item precludes purchase of any other) is assumed.

Of late, researchers studying multi-item purchasing have pursued one of three different approaches. First, the researcher can view the choice task as a number of separate singlecategory substitution problems (Andrews 2001). In this approach, the researcher carries out a simultaneous market structure analysis for the same consumers in each of several different product categories, and then compares parameter estimates across categories (cf. Ainslie and Rossi 1998). Applications of this approach to date have assumed that any correlation in purchase behavior across categories arises solely from consumer heterogeneity - that is, choice across categories is assumed to be independent for any given household.

A second approach to the modeling of multi-item purchasing has been to allow for joint purchasing of multiple items on a single shopping occasion. For example, Kamakura (2001) developed a multivariate tobit factor analysis representation of yogurt purchases that assumes the volume purchased of each item in the category is conditionally independent of all other purchases. (That is, any relationship among items arises from consumer preference heterogeneity and from non-stationarity of preferences, not from intra-consumer cross-item demand dependence.) In contrast, Petersen, Russell and Weiss (2001) developed a model in which each household allocates a budget across categories using parameters that vary with respect to household demographics. The outputs of both procedures are similar: a map in which consumers are placed near the cluster of items that they purchase. Products near one another in these maps are jointly purchased by households (either on one shopping trip or across a number of shopping trips). This type of representation is analogous to consideration set maps developed from pick-any survey data (Holbrook, Moore and Winer 1982). 
The third approach is to recast the multiple-item choice task as a product bundling problem. Multiple-item choice is modeled as a single choice from a conceivably very large set of plausible bundles of items. The multivariate probit model of Manchanda, Ansari and Gupta (1999) and the multivariate logistic model of Russell and Petersen (2000) use this approach in the context of market basket analysis. These models constitute internal market structure analyses because attributes of the categories are not assumed to be known a priori. In contrast, Rao and Chung (2001) propose a bundle choice model based upon the bundle utility specification developed by Farquhar and Rao (1976). Because their model requires that the attributes of bundle items are already known, it may be viewed as an external analysis. These models do not provide a market structure map. However they do demonstrate the viability of recasting multi-item purchasing of items as a problem of selecting a single bundle from a set of such bundles of items, which suggests that the market structure methods of single-item choice might be adapted to choice of single bundles.

\section{Promising Research Directions}

We began the paper with a one-sentence definition of market structure that suggested five desirable features of market structure models: they should (i) explain, (ii) be customerbased, and (iii) accommodate choice of multiple items and of bundles of items. They should also be able to (iv) go beyond the analysis of choice within a single, narrowlydefined, product category and (v) account for complementarity as well as substitutability. Our review of recent research included models that embody some of these features. We conclude with a brief discussion of research opportunities, emphasizing some we have touched upon and introducing others.

Market structure applies within, as well as among, customers. Many models of market structure assume that structure exists at the market level, due to customer heterogeneity, but does not exist for any individual customer. Yet there is good reason to believe that market structure exists at the individual level as well. For example, Yang, Allenby and Fennell (2002) incorporated data on both customer motives and consumption setting, and they found that both account for variation over time in customer evaluation of attribute importance and brand choice.

Data collection can be tailored to meet model requirements once these requirements are identified. The cost of collecting survey data is continually falling, and linking survey data to behavioral data is increasingly feasible. Can we discover generalities about market structure that could improve data collection? For example, can we develop a taxonomy of buyer purposes that affect market structure across many types of products and situations? Possible examples of such purposes might be (i) shopping for a gift rather than for oneself and (ii) choosing a brand when one's brand choice is socially conspicuous (e.g., automobiles) versus when it is not. Information relevant to many products could be collected routinely, 
which would increase the value of market structure analyses to companies and further our theoretical understanding of customer behavior.

Customers differ in their brand perceptions and in the accuracy of these perceptions. It is likely that customers differ in their perceptions of products (Chintagunta, Dube and Singh 2002) and are more certain about the attributes of some products than others (MacKay and Zinnes 1986; Erdem and Keane 1996). As we have seen, most models of market structure assume all customers perceive all products the same way and differ only in their evaluation of product attributes. Conditions favoring the validity of such an approximation need to be determined.

Many markets are dynamic and evolving rather than static. With few exceptions, current methods are more appropriate for categories and markets that are close to equilibrium. Many important markets do not exhibit this characteristic (e.g., high tech markets). Customers change their preferences as they acquire experience and in response to changing market offerings and communications. While periodic estimation of static market structure models can track changes, there is a need for models that can embody inherent market dynamics (e.g. Erdem and Keane 1996; Bronnenberg, Mahajan, and Vanhonacker 2000; Shocker, Bayus and Kim 2001).

Models should make fuller use of data indicative of market structure. Market structure is revealed in many ways: through perceived brand similarities, substitutability/ complementarity in use, cross-price elasticities (Cooper 1988), interpurchase times (Grover and Rao 1988), long-run budget allocation decisions (Petersen, Russell and Weiss 2001), and brand preference heterogeneity. Most models of market structure exploit only one of these indicators and ignore the others. The reliability of a market structure analysis, as well as user confidence in its validity, would be improved if it were based on several indicators rather than just one. Further investigations into the theoretical connections between different indicators of market structure, such as the link between cross-elasticities and brand switching probabilities discovered by Bucklin, Russell and Srinivasan (1998), would also be worthwhile.

Models must be comprehensible and possess high face validity or they won't be used. Managers find some indicators of market structure (such as perceived brand similarity) easier to understand and explain than others (such as brand preference heterogeneity). A model cannot be used as a basis for organizational decision-making unless it produces results that managers can verify using their understanding of a market.

Market structure analyses necessarily result in substantial uncertainty in parameter estimates. The degree of uncertainty needs to be communicated effectively to managers. Many models display only point estimates and simply list standard errors. If analyses are to improve decision-making, inferential uncertainty needs to be conveyed more effectively. Managers are generally risk-averse, and analyses involving exogenous variables can generate predictions that vary greatly in their precision. If managers are given adequate 
information about predictive uncertainty, they can favor decisions that correspond to more certain predictions or they can collect additional information to further reduce uncertainty.

There is a need for exploratory market structure analysis tools that can grapple autonomously with the very large, ill-structured and incomplete datasets now available. Many organizations have on hand huge customer datasets containing incomplete information on many behavioral and demographic variables. Market research suppliers of panel and market data can provide massive amounts of potentially useful information at ever-lower cost. Conventional models of market structure and common estimation techniques (maximum likelihood estimation, Markov chain Monte Carlo) cannot cope with these large datasets. Automated pattern recognition techniques, collectively known as data mining (Witten and Frank 2000), offer an attractive alternative in such settings. They can detect relationships among brands that lie outside conventional definitions of product category.

Models should be tailored to their intended use. Finally, no single market structure model can, nor probably should, include all of the features discussed here. More fullyfeatured models are inherently harder to estimate and explain and have greater data requirements. We should instead encourage development of an evolving portfolio of market structure models. Modelers wishing to contribute to this development would do well to tailor their model to the managerial decision task (Shankar 2001). Better tools for market structure analysis will help both managers and government regulators make better decisions through an improved understanding of the underlying determinants of product competition.

\section{References}

Andrews, Rick. (2001). "Inferring Market Structure from Customer Response to Competing and Complementary Products," Paper presented at the 2001 Invitational Choice Symposium, Pacific Grove, CA.

Ainslie, Andrew, and Peter E. Rossi. (1998). "Similarities in Choice Behavior Across Product Categories," Marketing Science, 17, 91-106.

Bacon, Lynd. (2001). "Perspectives on Internal Market Structure Analysis," Paper presented at the 2001 Invitational Choice Symposium, Pacific Grove, CA.

Bronnenberg, Bart, Vijay Mahajan, and Wilfried Vanhonacker. (2000). "The Emergence of Market Structure in New Repeat-Purchase Categories: The Interplay of Market Share and Retail Distribution," Journal of Marketing Research, 37, 16-31.

Bucklin, Randolf E., Gary J. Russell, and V. Srinivasan. (1998). "A Relationship Between Market Share Elasticities and Brand Switching Probabilities," Journal of Marketing Research, 35, 99-113.

Carroll, J. Douglas. (1972). "Individual Differences and Multidimensional Scaling," in R. N. Shepard, A. K. Romney, and S. B. Nerlove (eds.), Multidimensional Scaling: Theory and Applications in the Behavioral Sciences, Volume 1. New York and London: Seminar Press.

Chaturvedi, Anil, and J. Douglas Carroll. (1994). "An Alternating Combinatorial Optimization Approach To Fitting the INDCLUS and Generalized INDCLUS Models," Journal of Classification, 11, 155-170.

Chaturvedi, Anil, and J. Douglas Carroll. (2001). "Deriving Market Structure via Additive Decomposition of Market Shares," Paper presented at the 2001 Invitational Choice Symposium, Pacific Grove, CA. 
Chintagunta, Pradeep, Jean-Pierre Dube, and Vishal Singh. (2002). "Market Structure Across Stores: An Application of a Random Coefficients Logit Model with Store-Level Data." In Philip Hans Franses and Alan L. Montgomery (eds.), Econometric Models in Marketing: Advances in Econometrics, Volume 16. Amsterdam: JAI Press.

Cooper, Lee G. (1988). "Competitive Maps: The Structure Underlying Asymmetric Cross Elasticities," Management Science, 34, 707-723.

DeSarbo, Wayne, Ajay K. Manrai, and Lalita A. Manrai. (1993). "Non-Spatial Tree Models for the Assessment of Competitive Market Structure: An Integrated Review of the Marketing and Psychometric Literature," in Handbooks in Operations Research and Management Science, Volume 5: Marketing, Editors, J. Elaishberg and G. L. Lilien, New York: North-Holland.

DeSarbo, Wayne, and Vithala R. Rao. (1986). “A Constrained Unfolding Methodology for Product Positioning," Marketing Science, 5, 1-19.

Elrod, Terry. (1988). "Choice Map: Inferring a Product-Market Map From Panel Data," Marketing Science, 7, $21-40$.

Elrod, Terry. (1991). "Internal Analysis of Market Structure: Recent Developments and Future Prospects," Marketing Letters, 2, 253-266.

Elrod, Terry. (1997). "Obtaining Product-Market Maps from Preference Data," Proceedings of the 1997 Sawtooth Software Conference, Sequim, WA: Sawtooth Software, 273-288.

Elrod, Terry, and Gerald Häubl. (1998). "An Extended Random Coefficients Model, With Application to Metric Conjoint Analysis," Working Paper, University of Alberta School of Business.

Elrod, Terry, and Michael P. Keane. (1995). "A Factor-Analytic Probit Model for Representing the Market Structure in Panel Data," Journal of Marketing Research, 32, 1-16.

Erdem, Tulin, and Michael P. Keane. (1996). "Decision-Making Under Uncertainty: Capturing Dynamic Choice Processes in Turbulent Consumer Good Markets," Marketing Science, 15, 1-20.

Farquhar, Peter H., and Vithala R. Rao. (1976). "A Balance Model for Evaluating Subsets of Multiattributed Items," Management Science, 27, 528-539.

Grover, Rajiv, and Vithala R. Rao. (1988). "Inferring Competitive Market Structure Based on a Model of Interpurchase Intervals," International Journal of Research in Marketing, 5, 55-73.

Holbrook, Morris B., William L. Moore, and Russell S. Winer. (1982). "Constructing Joint Spaces from Pick-Any Data: A New Tool for Consumer Analysis," Journal of Consumer Research, 9, 99-105.

Johnson, Rich. (2001). "Positioning Thoughts for the Market Structure Workshop," Paper presented at the 2001 Invitational Choice Symposium, Pacific Grove, CA.

Kadiyali, Vrinda, Sudhir Karunakaran, and Vithala R. Rao. (2001). "Structural Analysis of Competitive Behavior: New Empirical Industrial Organization Methods in Marketing," International Journal of Research in Marketing, 18, 161-186.

Kamakura, Wagner A. (2001). "Modeling Multiple Quantity and Choice Decisions," Paper presented at the 2001 Invitational Choice Symposium, Pacific Grove, CA.

Lenk, Peter. (2001). "Hierarchical Bayes Factor Model Applied to Conjoint Analysis," Working Paper, University of Michigan Business School.

Lenk, Peter, et al. (1996). "Hierarchical Bayes Conjoint Analysis: Recovery of Partworth Heterogeneity From Reduced Experimental Designs," Marketing Science, 15, 173-191.

MacKay, David B., and Joseph L. Zinnes. (1986). "Probabilistic Model for the Multidimensional Scaling of Proximity and Preference Data," Marketing Science, 5, 325-344.

Manchanda, Puneet, Asim Ansari, and Sunil Gupta. (1999). "The 'Shopping Basket': A Model for MultiCategory Purchase Incidence Decisions," Marketing Science, 18, 95-114.

Martinetz, Th., and K. Schulten. (1994). "Topology Representing Networks," Neural Networks, 7, 507-522.

Mazanec, Josef. A., and H. Strasser. (2000). A Nonparametric Approach to Perceptions-Based Market Segmentation: Foundations. Vienna-New York: Springer.

Petersen, Ann, Gary J. Russell, and Doyle Weiss. (2001). "An Enriched Analysis of Multiple-Category Allocation Behavior," Working Paper, Tippie College of Business, University of Iowa.

Rao, Vithala R., and Jaihak Chung. (2001). "Issues in Modeling Consumers' Choice of Bundles of Multiattributed Items," Working Paper, Graduate School of Management, Cornell University. 
Ratneshwar, S. et al. (1999). "Product, Person, and Purpose: Putting the Consumer Back Into Theories of Dynamic Market Behavior,” Journal of Strategic Marketing, 7, 191-208.

Russell, Gary J., and Ann Petersen. (2000). "Analysis of Cross-Category Dependence in Market Basket Selection," Journal of Retailing, 76, 367-392.

Sawtooth Software, Inc. (1999). The CPM System for Composite Product Mapping. Sequim, WA: Sawtooth Software.

Shankar, Venkatesh. (2001). "Analyzing Purchases Among Multiple Categories and Sub-Categories," Paper presented at the 2001 Invitational Choice Symposium, Pacific Grove, CA.

Shocker, Allan D., Barry L. Bayus, and Namwoon Kim. (2001). "Product Complements And Substitutes in a Dynamic World: The Relevance Of 'Other Products',' Working Paper, College of Business, San Francisco State University.

Yang, Sha, Greg M. Allenby, and Geraldine Fennell. (2002). "Modeling Variation in Brand Preference: The Roles of Objective Environment and Motivating Conditions," Marketing Science, 21, 14-31.

Witten, Ian, and Eibe Frank. (2000). Data Mining: Practical Machine Learning Tools and Techniques with Java. New York: Morgan Kaufmann Publishers.

Wittenschlaeger, Thomas M., and John A. Fiedler. (1997). "Current Practices in Perceptual Mapping," Proceedings of the 1997 Sawtooth Software Conference. Sequim, WA: Sawtooth Software, 259-270. 